\title{
Study of annealing effects in In-Sb bilayer thin films
}

\author{
R K MANGAL ${ }^{\dagger}$ and Y K VIJAY* \\ Department of Physics, University of Rajasthan, Jaipur 302 004, India \\ ${ }^{\dagger}$ Jaipur Engineering College and Research Centre, Jaipur 303 905, India \\ MS received 16 November 2006
}

\begin{abstract}
The thin films of In-Sb having different thicknesses of antimony keeping constant thickness of indium was deposited by thermal evaporation method on ITO coated conducting glass substrates at room temperature and a pressure of $10^{-5}$ torr. The samples were annealed for $1 \mathrm{~h}$ at $433 \mathrm{~K}$ at a pressure of $10^{-5}$ torr. The optical transmission spectra of as deposited and annealed films have been carried out at room temperature. The variation in optical band gap with thickness was also observed. Rutherford back scattering and X-ray diffraction analysis confirms mixing of bilayer system. The transverse $I-V$ characteristic shows mixing effect after annealing at $433 \mathrm{~K}$ for $1 \mathrm{~h}$. This study confirms mixing of bilayer structure of semiconductor thin films.
\end{abstract}

Keywords. InSb; thin films; transmission; band gap; RBS; $I-V$ characteristics; XRD.

\section{Introduction}

Indium antimonide ( $\mathrm{InSb})$ is one of the most widely studied III-V group compound semiconductors. It is an important material in the field of infrared detectors in the $3-5 \mu \mathrm{m}$ wavelength range due to its high electron hole mobility and low energy gap at room temperature (Chen et al 1992). It is also used for the fabrication of high speed Hall and optoelectronic devices (Milnes 1973; Asauskas et al 1980; Branski et al 1990). The deposition of indium antimonide thin films is more suitable for fabricating photo-electronic (Osborn 1984), magneto-electric (Oi et al 1978) conversion devices and antenna-coupled infrared detectors (Yasuoka et al 1978). Thin films of InSb may be deposited by different methods like r.f. sputtering (Milyazaki et al 1991), MBE (Chyi et al 1989), CVD (Chang and Bedair 1985), flash evaporation (Carroland and Spivak 1966) and vacuum evaporation (Asauskas et al 1980). The stack elemental layer deposition method has been used for CdTe by Cruz and de Avillez (2000), copper indium diselenide thin films by Carter et al (1987), ZnSe and InSb thin films by Singh and Vijay (2004) and AlSb thin films by Singh and Vijay (2005). It is particularly suitable for deposition of compound semiconductor thin films, as it provides good control of composition (Singh and Vijay 2004). The importance of this paper is to understand the inter-diffusion reaction of bilayer structure in a controlled manner.

In this paper, we are presenting the method of preparation of semiconducting thin films and characterization by optical transmission spectra, Rutherford back scattering spectrometry, $I-V$ characteristics and X-ray diffraction patterns.

*Author for correspondence (yk_vijay@sancharnet.in)

\section{Experimental}

\subsection{Sample preparation}

The high purity indium $(99.99 \%)$ powder and pure antimony (99.5\%) have been obtained from BDH Chemicals Ltd., Pool, England, to prepare In-Sb bilayer thin films. Bilayer structure of In-Sb was deposited onto ITO coated conducting glass substrates by thermal evaporation method at a pressure of $10^{-5}$ torr using HIND HI VACUUM unit. We deposited indium and antimony films one by one on ITO coated conducted glass substrates. The glass substrates were placed in the substrate holder above the boats carrying materials. The first material evaporated was antimony and later indium to prepare bilayer of InSb thin films. The thickness of indium layer was $3000 \AA$ on each substrate over antimony layer having thicknesses $1000 \AA$, $1500 \AA$ and $2000 \AA$, respectively. Thickness of the samples was measured by digital quartz crystal thickness monitor.

\subsection{Heat treatment}

The samples were annealed in vacuum at a pressure of $10^{-5}$ torr for $1 \mathrm{~h}$ at $433 \mathrm{~K}$ temperature using HIND HI VACUUM chamber.

\subsection{Absorption spectra}

The transmission spectra of as deposited and diffused samples were recorded using Systronics spectrophotometer, model - 117 . 


\subsection{Rutherford backscattering spectra}

The RBS data have been recorded at the Institute of Physics, Bhubaneswar, using the Pelletron facility with alpha particle beam $\left(\mathrm{He}^{2+}\right)$ of $3 \mathrm{MeV}$. The back-scattered alpha particles were recorded at an angle of $160^{\circ}$ using a surface barrier detector.

\subsection{X-ray diffraction spectra}

The X-ray diffraction patterns of as deposited and vacuum annealed samples have been carried out using Philips PW 1730 model having source, $\mathrm{CuK} \alpha, \mathrm{XRD}$ of wavelength, $1.5472 \AA$ set up.

\section{$2.6 I-V$ characteristics}

Transverse $I-V$ characteristics of as deposited and vacuum annealed films were recorded using Keithley-238 high current source measuring unit.

\section{Results and discussion}

\subsection{Optical properties}

Figures 1-2 show transmission (\%) vs wavelength spectra of as deposited and annealed In-Sb bilayer thin films. The increase in optical transmission is related to the increase in grain size of the films with increasing substrate temperature (Holloway and Sinclair 1987). In annealed films the optical absorption edge produced by transitions between two state densities, both of which rapidly change as a function of energy, corresponds to the valence and conduction band edges for the ideal stable continuous random network structure as proposed by Connell et al

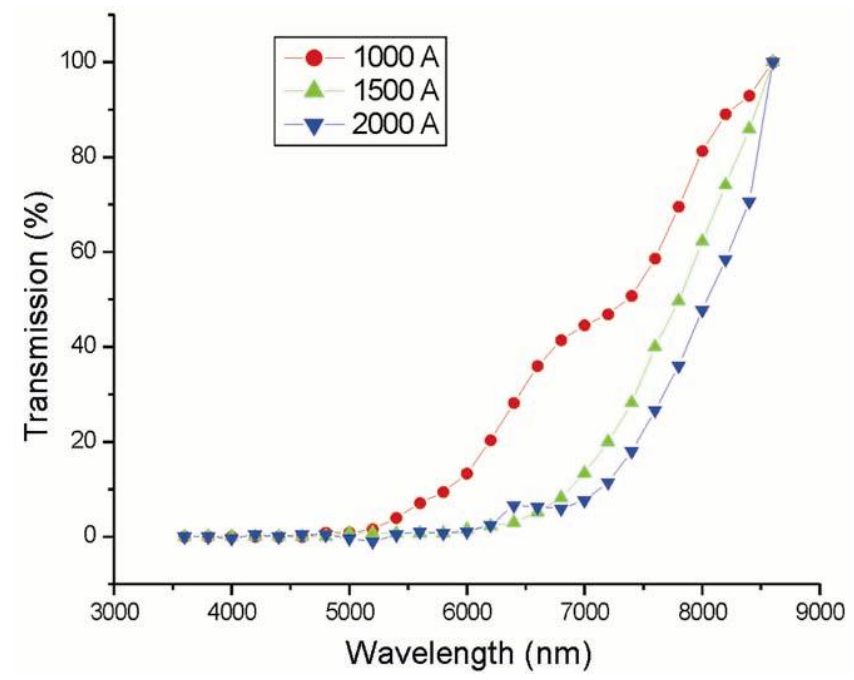

Figure 1. Wavelength vs transmission spectrum of as deposited samples.
(1972). The optical band gap of these films was calculated using the famous Tauc (1974) relation

$$
\alpha h v=A(h v-E g)^{n},
$$

where $h v$ is photon energy, $\alpha$ the absorption coefficient, $E g$ the band gap, $A$ a constant, $n=0.5$ for direct band gap material, $n=2$ for indirect band gap material. Since In$\mathrm{Sb}$ is a direct band gap material, so in the present work, we have used $n=0.5$ and the intercept of straight line to energy axis $(\alpha h v)^{2}=0$ used to find out the optical band gap. It is observed that the value of optical band gap decreases with thickness keeping constant temperature $(433 \mathrm{~K})$ in all films. It may be due to change in the inter-diffusion ratio of In and $\mathrm{Sb}$ interface at different thicknesses. In our case, optical band gap was found to change from $0 \cdot 169$ $0.153 \mathrm{eV}$ (table 1). Shift in band gap of annealed films was due to variation of concentration in bilayer and increasing the grain size of films with temperature (Singh and Vijay 2004).

\subsection{Rutherford back scattering analysis}

Figures 3-5 show the channel no. vs counts spectra in which peaks of annealed films are shifted downwards, because alpha particles pass in only indium layer in as deposited films

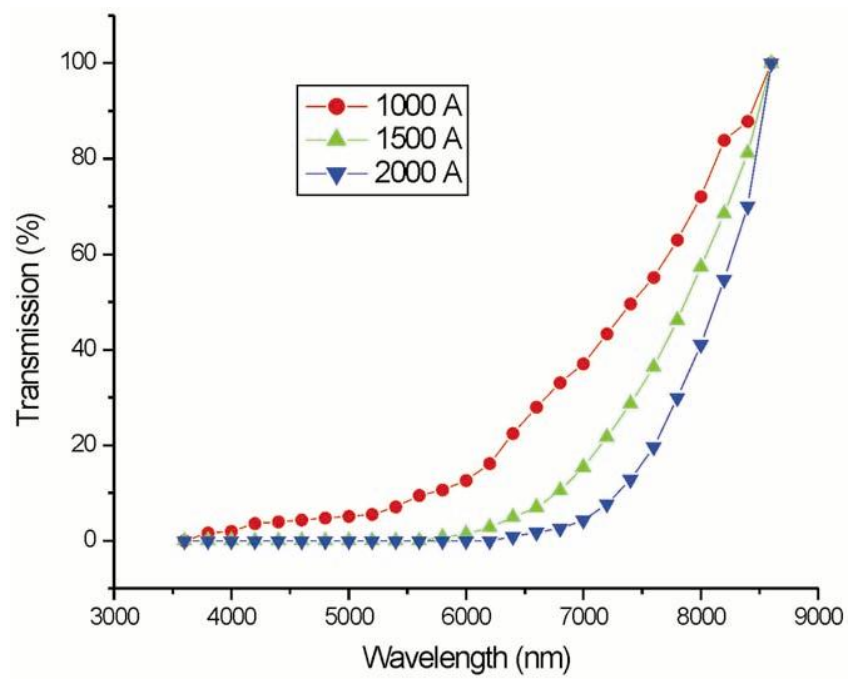

Figure 2. Wavelength vs transmission spectrum of annealed samples.

Table 1. Variation of optical band gap with thickness of annealed films.

\begin{tabular}{lc}
\hline Thickness of In-Sb $(\AA)$ & Band gap of annealed film $(\mathrm{eV})$ \\
\hline $3000-1000$ & $0 \cdot 169$ \\
$3000-1500$ & $0 \cdot 162$ \\
$3000-2000$ & $0 \cdot 153$ \\
\hline
\end{tabular}


which is a lighter nuclei element compared to antimony but in vacuum annealed films particles pass from mixed (InSb) material. The energy loss in vacuum annealed films is higher compared to as deposited samples, that confirms

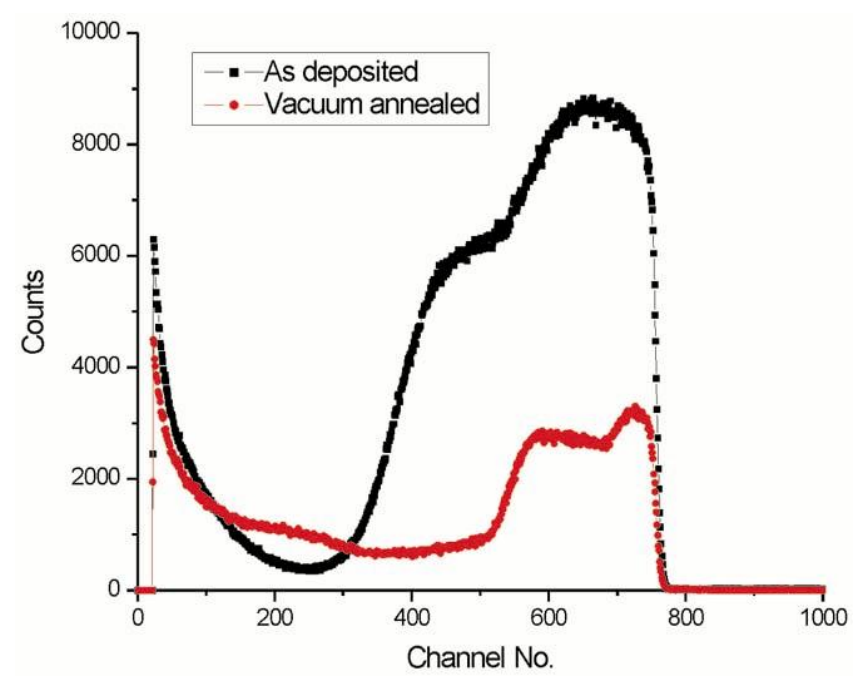

Figure 3. Rutherford back scattering spectrum of as deposited and vacuum annealed InSb bilayer thin film of thickness, 3000$1000 \AA$.

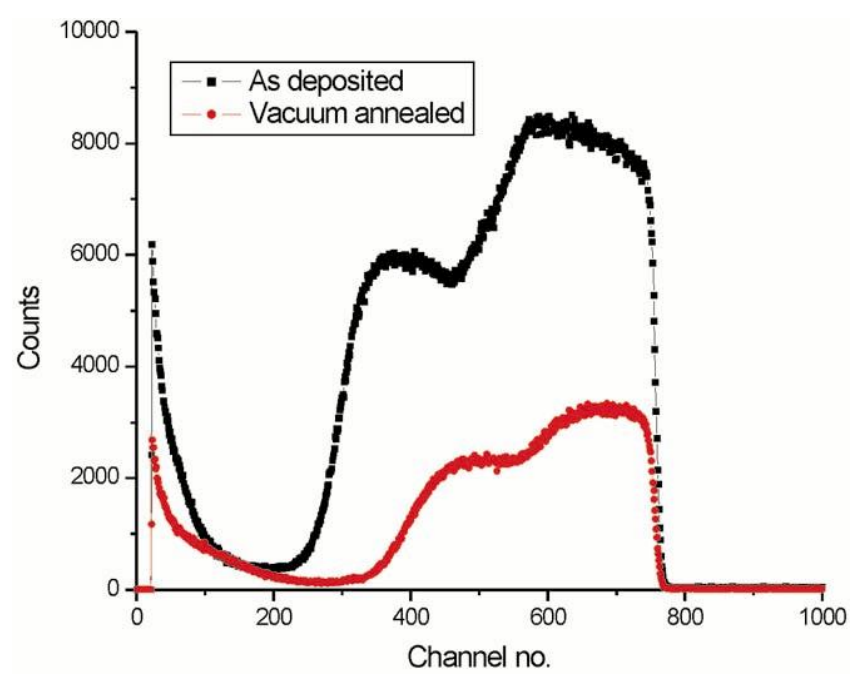

Figure 4. Rutherford back scattering spectrum of as deposited and vacuum annealed InSb bilayer thin film of thickness, 3000$1500 \AA$.

Table 2. Variation of resistance of as deposited and vacuum annealed In-Sb bilayer thin films.

\begin{tabular}{lccc}
\hline $\begin{array}{l}\text { S1. } \\
\text { no. }\end{array}$ & $\begin{array}{c}\text { Thickness of } \\
\text { In-Sb films }(\AA)\end{array}$ & $\begin{array}{c}\text { Resistance of as } \\
\text { deposited films }(\Omega)\end{array}$ & $\begin{array}{c}\text { Resistance of vacuum } \\
\text { annealed films }(\Omega)\end{array}$ \\
\hline 1 & $3000-1000$ & 20 & 15 \\
2 & $3000-1500$ & 15 & 10 \\
3 & $3000-2000$ & 19 & 14 \\
\hline
\end{tabular}

the inter-diffusion of bilayer structure of In and Sb. Figures 3 and 4 show better mixing compared to figure 5 . It is also justified by our optical band gap measurements. Figure 5 shows that the effect of antimony thickness dominated. It means inter-diffusion occurs between the In/Sb bilayer. These RBS data also agree with recent work of temperature annealing of In-Sb, $\mathrm{Zn}-\mathrm{Se}$ and Ti-Si thin films (Holloway and Sinclair 1987; Singh and Vijay 2004, 2005) and suggested that annealing process helped in inter-diffusion of the elemental layer with temperature.

\subsection{I-V characteristics}

Figures 6-8 show plotted graph of transverse currentvoltage $(I-V)$ characteristics for thicknesses $3000-1000 \AA$,

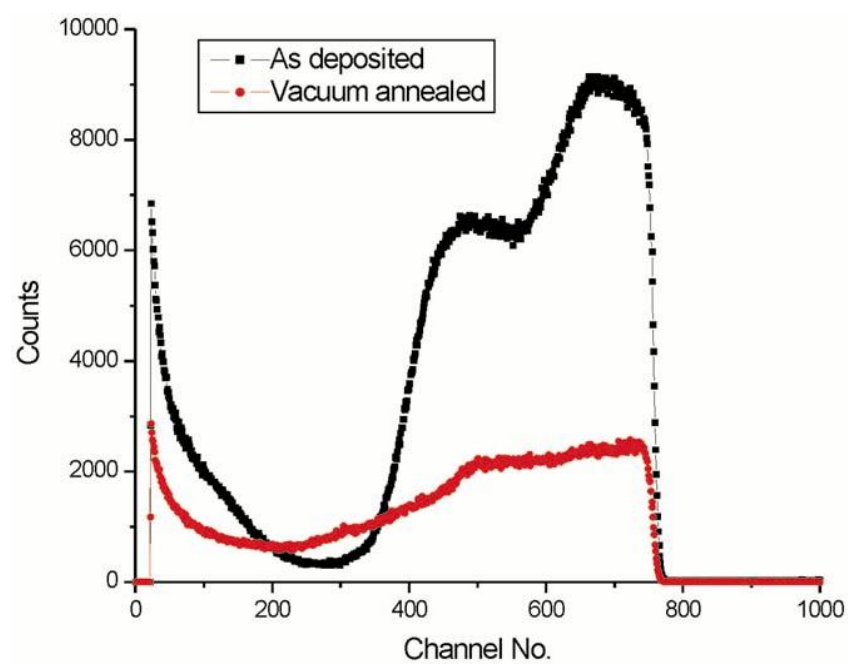

Figure 5. Rutherford back scattering spectrum of as deposited and vacuum annealed $\mathrm{In}-\mathrm{Sb}$ bilayer thin film of thickness, $3000-2000 \AA$

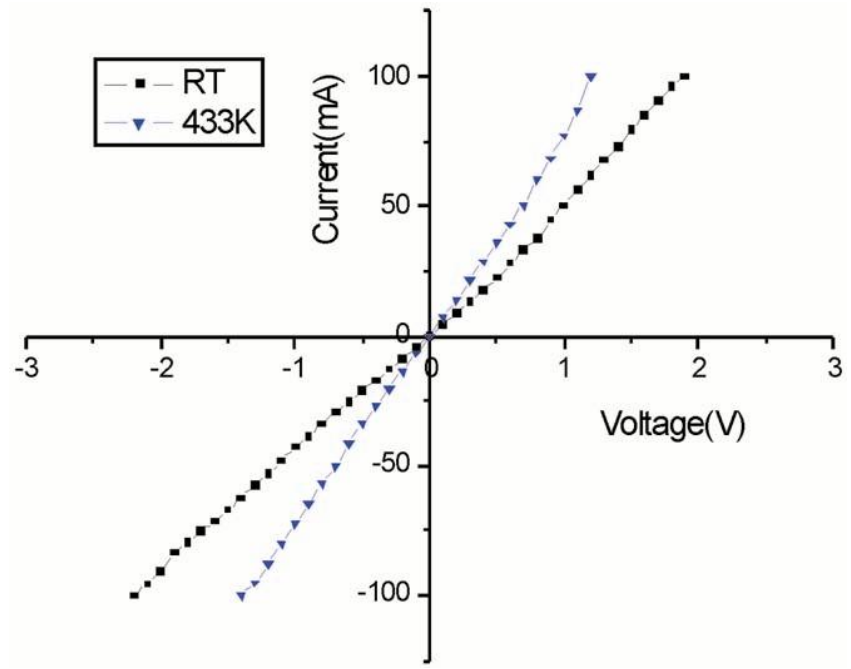

Figure 6. $I-V$ characteristics of the $\mathrm{In}-\mathrm{Sb}$ thin film having thickness, 3000-1000 § 


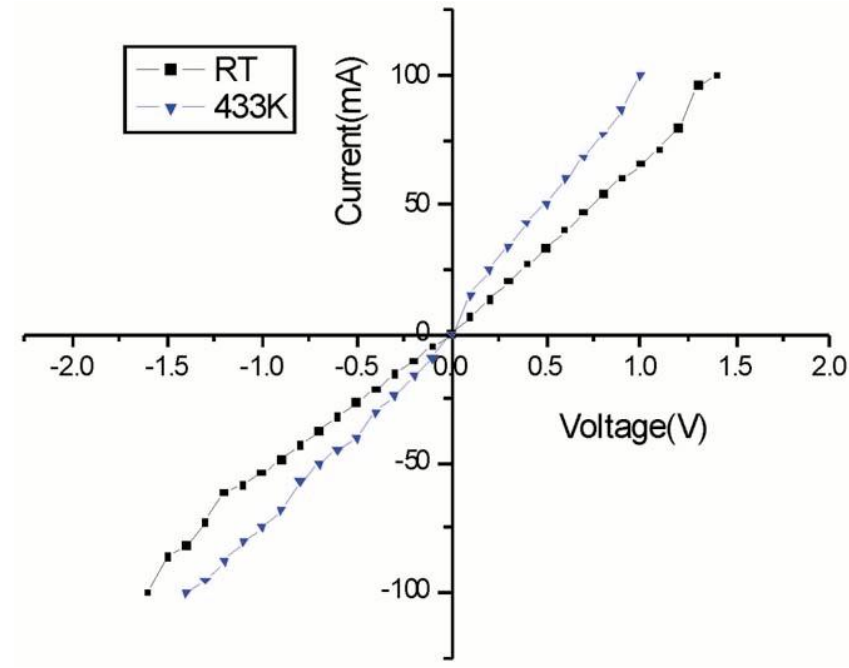

Figure 7. $I-V$ characteristics of the $\mathrm{In}-\mathrm{Sb}$ thin film having thickness, $3000-1500 \AA$.

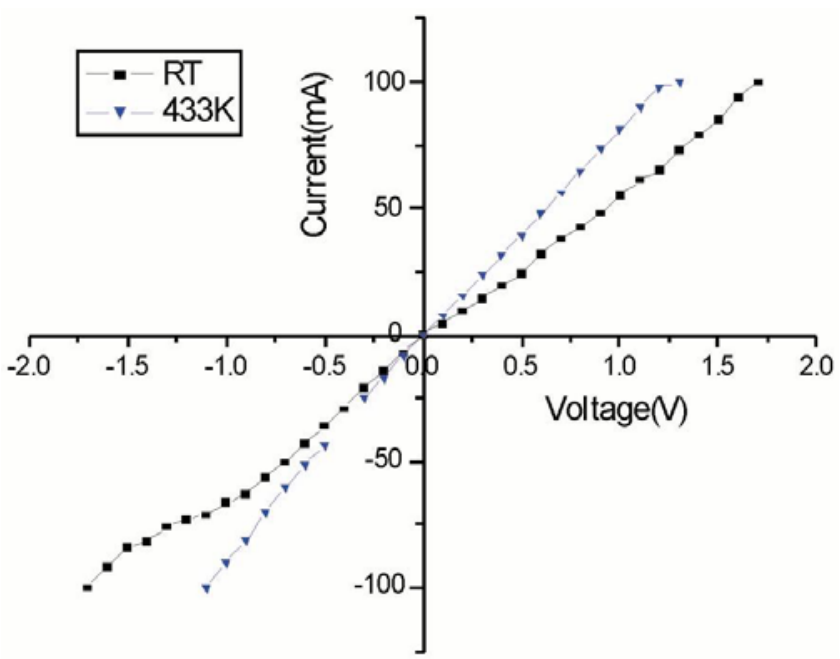

Figure 8. $I-V$ characteristics of the $\mathrm{In}-\mathrm{Sb}$ thin film having thickness, 3000-2000 A.

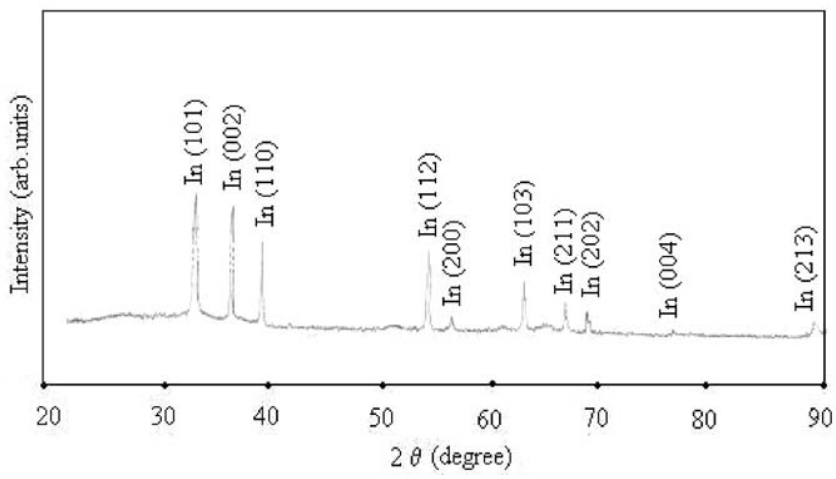

Figure 9. X-ray diffraction pattern of as deposited In-Sb bilayer thin film having thickness, 3000-1500 §.
$3000-1500 \AA$ and $3000-2000 \AA$, respectively of In-Sb bilayer thin films.

In these figures we have observed that after annealing up to $433 \mathrm{~K}$ for $1 \mathrm{~h}$, In-Sb films show low resistance compared to as deposited films due to mixing of In-Sb bilayers. Numerical values are shown in table 2. This is due to mixing of both films at this temperature by diffusion process and mobility of electrons increases in the mixed material. So it is clear that the resistivity of material after annealing at $433 \mathrm{~K}$ increases, conductivity decreases. Similar effect has been observed in the case of In-Sb bilayer thin films by Singh and Vijay (2005).

\subsection{X-ray diffraction analysis}

The XRD analysis is a non-destructive probe to study the compositional variation of the elements in compound semiconductors. The XRD patterns plotted in the range $20-90^{\circ}(2 \theta)$ vs intensity (arbitrary units) are shown in figures 9-10. XRD pattern of as grown sample shows several peaks of indium, but in the vacuum annealed sample, it shows peaks of In-Sb alloy as well as indium. These peaks indicate the formation of In-Sb phase and improvement of grain size in thin film structure. We found zinc blend structure $(a=b=c)$ of formed InSb compound semiconductor. From the formula

$$
d=a / \sqrt{ } h^{2}+k^{2}+l^{2},
$$

where $a$ is atomic radius of the compound semiconductor, $h, k$ and $l$ are planes. Observed $d$-values and standard $d$ -

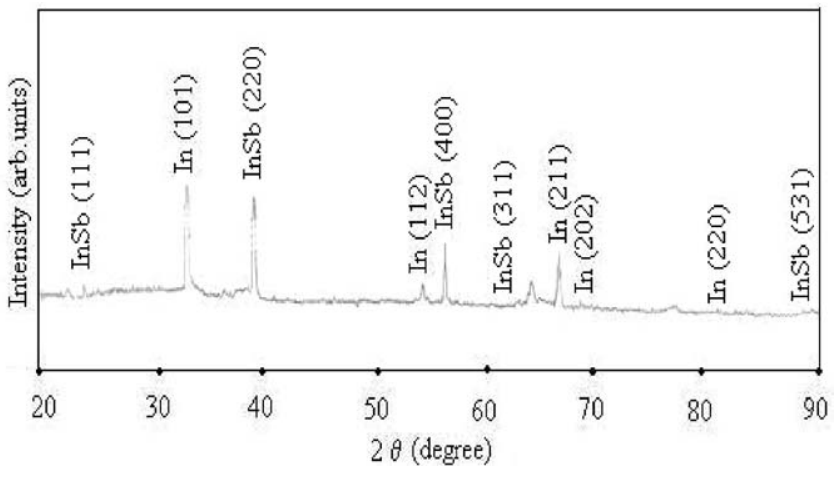

Figure 10. X-ray diffraction pattern of vacuum annealed InSb bilayer thin film having thickness, 3000-1500.

Table 3. Observed and standard $d$-values of vacuum annealed In-Sb thin film.

\begin{tabular}{lcccc}
\hline $\begin{array}{l}\text { S1. } \\
\text { no. }\end{array}$ & $2 \theta$ (degree) & $h k l$ planes & $\begin{array}{c}\text { Observed } \\
d \text {-values }(\AA)\end{array}$ & $\begin{array}{c}\text { Standard } \\
d \text {-values }(\AA)\end{array}$ \\
\hline 1 & 24 & $(111)$ & 3.739 & 3.74 \\
2 & 38 & $(220)$ & 2.287 & 2.290 \\
3 & 64 & $(311)$ & 1.9511 & 1.953 \\
4 & 89 & $(531)$ & 1.0936 & 1.095 \\
\hline
\end{tabular}


values (from ASTM cards) of vacuum annealed In-Sb thin film with their planes are shown in table 3.

Similar effect has been observed by Cruz and de Avillez (2000) in the case of CdTe, ZnSe (Singh and Vijay 2004) and InSb (Singh and Vijay 2005). Present observed results are in good agreement with XRD pattern observed by Masaaki et al (1991) for In-Sb with variation of substrate temperature. In the present study, we annealed the InSb bilayer structure and found the XRD pattern as shown in figure 10. Some peaks of indium tin oxide (ITO) are deleted from these XRD data. Present work is in full agreement with literature (Wider and Clawson 1965; Okinura et al 1989).

\section{Conclusion}

From the above detailed study of this material, we conclude that vacuum annealing may be used for mixing the bilayer structure of In and $\mathrm{Sb}$, because our optical band gap data, $\mathrm{XRD}$ analysis, RBS analysis as well as $I-V$ characteristics show formation of In-Sb compound semiconductor.

\section{Acknowledgements}

Authors are highly thankful to Dr B R Shekhar, Institute of Physics, Bhubaneswar, for providing RBS facility, and Dr Anwar Rais, Geological Survey of India, Jaipur, for providing XRD facility for characterization of our samples. We are also thankful to Balram Tripathi and Ankur Jain, University of Rajasthan, Jaipur, for help in sample preparation.

\section{References}

Asauskas R, Dobro V and Krotkus A 1980 Sov. Phys. Semicond. 141377

Branski P I, Gorodrichi O P and Shevchenko V V 1990 Infrared Phys. 3059

Carrolnand J A and Spivak J F 1966 Solid State Electron. 9383

Carter M J, Anson I I, Knowles A and Hill R 1987 Proceedings of the 19th IEEE photovoltaic specialists conference (New Orleans: IEEE, NY) p. 1275

Chang P K and Bedair S M 1985 Appl. Phys. Lett. 46383

Chen L P, Luo J J, Liu R H, Yang S P and Pang Y M 1992 Jpn. J. Appl. Phys. Lett. 31813

Chyi J A, Biswanas D, Iyer S, Kumar V and Hmorkoe N S 1989 Appl. Phys. Lett. $\mathbf{5 4} 1016$

Connell G A N and Willian P 1972 J. Non-cryst. Solids 8215

Cruz L R and de Avillez R R 2000 Thin Solid Films 37315

Holloway K and Sinclair R 1987 J. Appl. Phys. 461

Masaaki Isai, Beevens J and Cheeke J D N 1991 J. Appl. Phys. 1069

Milnes A G 1973 Deep impurities in semiconductors (New York: Wiley) p. 45

Milyazaki T, Mori M and Adachi S 1991 Appl. Phys. Lett. 58 116

Oi T, Kotera N, Shigeta J, Yamamoto N and Nakashima N 1978 Jpn J. Appl. Phys. 17407

Okinura H, Matsume T and Ohshita M 1989 J. Appl. Phys. 966 Osborn G C 1984 J. Vac. Sci. Technol. B2 176

Singh M and Vijay Y K 2004 Appl. Surf. Sci. 23979

Singh M and Vijay Y K 2005 Indian J. Pure \& Appl. Phys. 43383

Tauc J (ed.) 1974 Amorphous and liquid semiconductors (New York: Plenum) p. 159

Wider H H and Clawson A R 1965 Solid State Electron. 8467 Yasuoka Y, Okuda T and Inoue N 1978 J. Appl. Phys. 17407 